\title{
Krüppel-like factor 12 is a novel negative regulator of forkhead box 01 expression: a potential role in impaired decidualization
}

Hui Zhang ${ }^{1 \dagger}$, Xudong Zhu ${ }^{2 \dagger}$, Jing Chen ${ }^{3+}$, Yue Jiang ${ }^{3}$, Qun Zhang ${ }^{3}$, Chengcai Kong ${ }^{3}$, Jun Xing ${ }^{3}$, Lijun Ding ${ }^{3}$, Zhenyu Diao ${ }^{3}$, Xin Zhen ${ }^{3}$, Haixiang Sun ${ }^{1 *}$ and Guijun Yan ${ }^{3^{*}}$

\begin{abstract}
Background: Decidualization is a prerequisite for successful implantation and the establishment of pregnancy. Krüppel-like factor 12 (KLF12) is a negative regulator of endometrial decidualization in vitro. We investigated whether KLF12 was associated with impaired decidualization under conditions of repeated implantation failure (RIF).

Methods: Uterine tissues were collected from a mouse model of early pregnancy and artificial decidualization for immunohistochemistry, Western blot and real-time PCR analysis. Reporter gene assays, chromatin immunoprecipitation-PCR and avidin-biotin conjugate DNA precipitation assays were performed to analyze the transcriptional regulation of forkhead box O1 (FOXO1) by KLF12. Furthermore, the protein levels of KLF12 and FOXO1 in patients with RIF were analyzed by Western blot and immunohistochemistry.

Results: KLF12 led to defective implantation and decidualization in the mouse uterine model of early pregnancy and artificial decidualization by directly binding to the FOXO1 promoter region and inhibiting its expression in human endometrial stromal cells. Elevated KLF12 expression was accompanied by decreased FOXO1 expression in the endometria of patients with RIF.

Conclusions: As a novel regulator, KLF12 predominantly controls uterine endometrial differentiation during early pregnancy and leads to implantation failure.
\end{abstract}

Keywords: KLF12, FOXO1, Decidualization, RIF

\section{Background}

Stromal decidualization in the endometrium is crucial for successful embryo implantation and the maintenance of pregnancy. This process is defined by the mesenchymalto-epithelial transformation of endometrial fibroblasts and stromal cells into secretory epithelioid decidual cells, which affect endometrial receptivity [1]. In mice and rats, mechanical or chemical stimulation of the endometrium is required to induce decidual transformation. In contrast with what occurs in many species, decidualization of the

\footnotetext{
*Correspondence: stevensunz@163.com; yanguijun55@163.com

${ }^{\dagger}$ Equal contributors

${ }^{1}$ Reproductive Medicine Center, Drum Tower Clinic Medical College of Nanjing Medical University, Nanjing 210029, Jiangsu, China

${ }^{3}$ Department of Obstetrics and Gynecology, Reproductive Medicine Center, Nanjing Drum Tower Hospital, Nanjing University Medical School, Nanjing 210008, Jiangsu, China

Full list of author information is available at the end of the article
}

human endometrial stroma begins spontaneously during the late luteal phase of the menstrual cycle, even in the absence of a conceptus [2]. Regardless of the mechanism of initiation, endometrial decidualization is maintained in an embryo-dependent manner. A variety of pregnancy disorders, including infertility, recurrent pregnancy loss (RPL) [3], implantation failure [4], utero-placental disorders, endometriosis [5] and pre-eclampsia [6], have been associated with impaired decidualization.

The steroid hormones estrogen and progesterone, endometrial autocrine and paracrine factors, and miRNAs have been implicated in decidualization, although how these molecules coordinate an endometrial response remains unclear $[7,8]$. In addition to nuclear receptors (e.g., progesterone receptor (PR)), some transcription factors have been identified as critical regulators of decidualization, including homeobox transcription factors [9], CCAAT/enhancer 
binding protein- $\beta(C / E B P \beta)[10]$, and members of the $O$ subclass of the forkhead family of transcription factors (FOXOs) [11]. Sun et al. reported that a zinc fingercontaining transcription factor, Krüppel-like factor 5 (KLF5), is expressed dynamically in the luminal epithelium and stroma throughout peri-implantation. KLF5 regulates formation and development of the trophectoderm, inner cell mass, and primitive endoderm, and $\mathrm{KLF5}^{-/-}$mice display embryonic lethality due to developmental defects in the preimplantation embryo. Conditional deletion of KLF5 in the uterus compromises implantation and decidualization [12]. To date, many KLFs, such as KLF12, have been found to participate in steroid hormone signaling and to exhibit pleiotropic actions in uterine endometrial cells [1315]. KLF12 has been shown to bind to the promoter regions of target genes and represses their expression through an N-terminal PVDLS sequence (Pro-Xaa-Asp-Leu-Ser) that promotes physical interaction with $\mathrm{CtBP}$ co-repressors [16]. We have previously demonstrated that KLF12 is expressed in the glandular epithelium (GE) and stromal cells of secretory phase endometrial tissue and that it negatively regulates endometrial decidualization and marker gene transcription [14].

In this study, we showed that KLF12 impaired embryo implantation and endometrial decidualization in mice and that it regulated FOXO1 expression by binding to a CAGTGGG element within the FOXO1 promoter. Moreover, KLF12 was aberrantly expressed in midsecretory endometrial samples from patients with repeated implantation failure (RIF).

\section{Methods}

\section{Animals and treatments}

ICR mice were purchased from the Laboratory Animal Center of Yangzhou University (Yangzhou, China). All animals were bred at the Laboratory Animal Center of Nanjing Drum Tower Hospital (Nanjing, China). All studies were approved by the Institutional Animal Care and Use Committee of Nanjing Drum Tower Hospital (SYXK 2009-0017).

Females (six weeks old) were mated with fertile males, and the morning on which a vaginal plug was observed was designated as day 0.5 of pregnancy. Twenty microliters $\left(2 \times 10^{8} \mathrm{TU} /\right.$ side $)$ of Ad-LacZ or Ad-Flag-KLF12 adenovirus (packaged, amplified and purified as previously reported [14]) was injected into the bilateral uterine horns of the mice in the morning on 1.5 days post-coitus (dpc). Then, the mated mice were intravenously injected with Chicago Sky Blue 6B (Sigma, St. Louis, MO, USA) in the afternoon on $4.5 \mathrm{dpc}$ to evaluate the state of implantation (Fig. 1a).

Artificial decidualization was performed as described previously [17]. Briefly, six-week-old mice were subjected to bilateral ovariectomy and treated with $20 \mu \mathrm{L}$ of
Ad-Flag-KLF12 or Ad-LacZ via injection into the bilateral uterine horns $\left(2 \times 10^{8} \mathrm{TU} / \mathrm{side}\right)$. Ten days later, the mice received intravenous injection of $20 \mu \mathrm{L}$ adenovirus $\left(2 \times 10^{8} \mathrm{TU} /\right.$ mouse $)$. After two days of rest, the mice were treated with a regimen of E2 and P4 as per the standard protocol and injected with sesame oil into the uterine horns as a decidualization stimulus (Fig. 2a).

\section{Endometrial sampling and cell culture}

All endometrial samples were obtained via endometrial biopsy between days 19 and 23 of the menstrual cycle from 29 patients who exhibited regular menstrual cycles (28-31 days) and who were not taking any oral contraceptives. The demographic details of the patients are summarized in Table 1. RIF was defined as the failure to achieve pregnancy following a minimum of three fresh or frozen cycles, during which at least four good-quality embryos were transferred to the uterus [18]. The subjects in the control group had undergone no less than one successful cycle and delivery. This study was approved by the Institutional Review Board of Nanjing Drum Tower Hospital.

Human endometrial stromal cells (hESCs) were isolated and cultured as described previously, and the purity of the cultured stromal cells was determined by cell immunohistochemistry, as shown in Additional file 1: Figure S1. Decidualization was induced according to a previously published protocol $[19,20]$.

\section{Western blotting}

Proteins were prepared as previously described [19], and the protein content was measured using the Bradford assay (Bio-Rad Laboratories, Hercules, CA, USA). Equal amounts $(20 \mu \mathrm{g})$ of protein were separated by SDSPAGE, and immunoblotting was performed with primary antibodies against FOXO1 (Cell Signaling Technology, Danvers, MA, USA, 1:1000), KLF12 (Santa Cruz Biotechnology, CA, USA, 1:2000) or GAPDH (Bioworld Technology, MN, USA, 1:10000), followed by incubation with a horseradish peroxidase (HRP)-conjugated secondary antibody and Flag-HRP (Sigma, 1:5000). The bands were detected using an enhanced chemiluminescence kit (Amersham Biosciences Corp., Piscataway, NJ, USA), and densitometric analysis of each band was performed with Quantity-one (Bio-Rad) software.

Quantitative reverse transcription real-time PCR (qRT-PCR) Total RNA was extracted from primary hESCs and from the mouse uterine and human endometrial samples. RNA was reverse-transcribed and subjected to qRT-PCR analysis. The primers for qRT-PCR were as follows: mPRP, sense 5'-GAGAATGGCTGCTCAGATCC-3', antisense 5'-GTTCAGGTCCATGAGCTGGT-3'; mFOXO1, sense 5'-TCGTACGCCGACCTCATCA-3', antisense 5' -CTG 


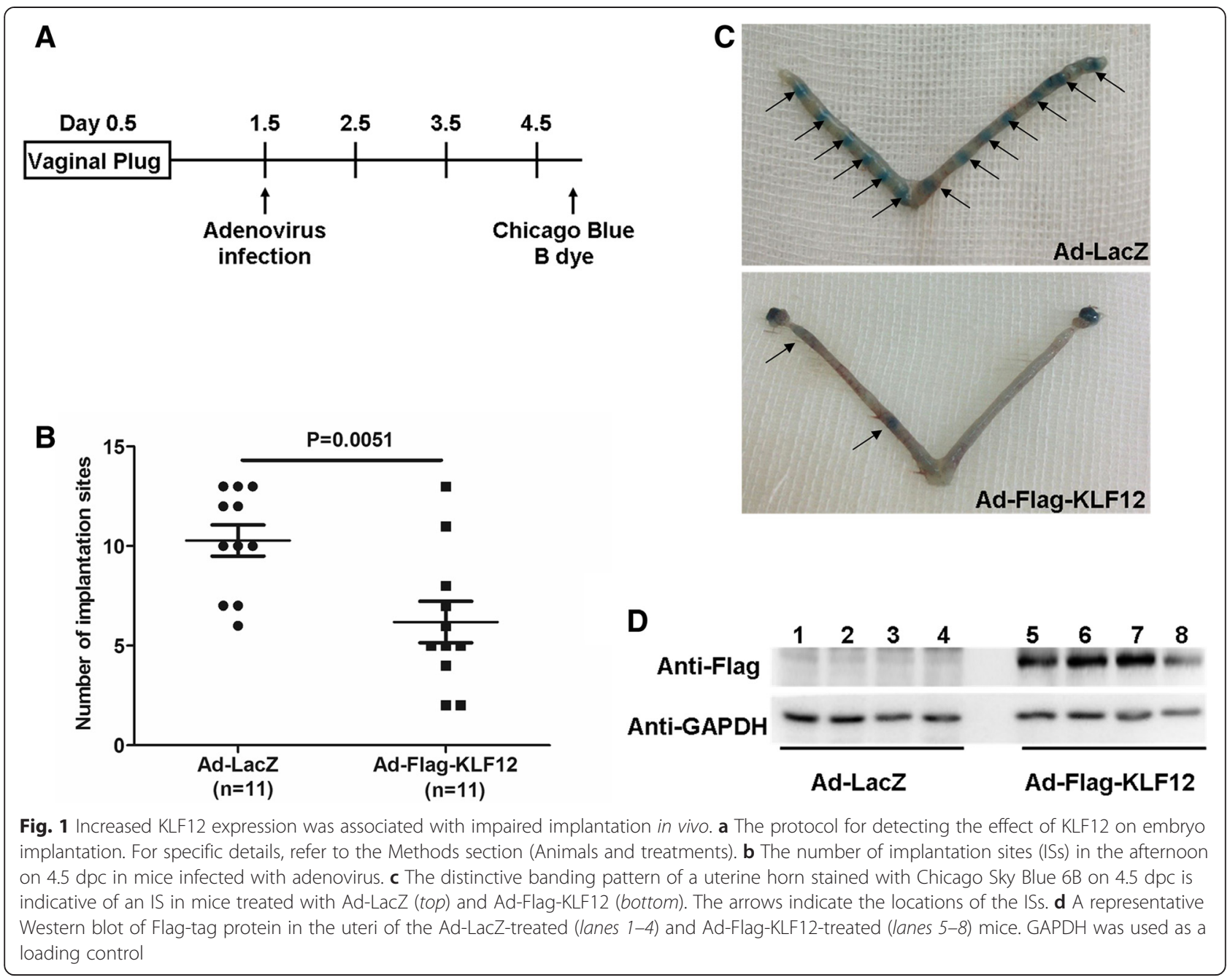

TCGCCCTTATCCTTGAAGT-3'; hKLF12, sense 5'-CC TTTCCATAGCCAGAGCAG-3', antisense 5'-TTGCA TCCCTCAAAATCACA-3'; hFOXO1, sense 5'-TCAT GTCAACCTATGGCAG-3', antisense 5' -CATGGTGCTTACCGTGTG-3'; m18S rRNA, sense 5'-CGGACATCTAAGGGCATCAC-3', antisense 5'-ATGGCCGTTCT TAGTTGGTG-3'; and h18S rRNA, sense 5'-CGGCTACCACATCCAAGGAA-3', antisense 5'-CTGGAATTACC GCGGCT-3'. The qRT-PCR data were analyzed using the $2^{-\triangle \Delta C T}$ method, with $18 \mathrm{~S}$ rRNA as an internal control.

\section{Immunostaining}

After the samples were dewaxed, endogenous peroxidase activity was blocked using freshly prepared phosphatebuffered saline (PBS) containing $0.3 \%$ hydrogen peroxide for $15 \mathrm{~min}$. Antigen retrieval was conducted by autoclaving the samples at $121{ }^{\circ} \mathrm{C}$ for $15 \mathrm{~min}$ in the presence of EDTA ( $\mathrm{pH}$ 9.0). The sections were washed with PBS and then incubated with antibodies against KLF12 (1:600 dilution, Santa Cruz Biotechnology) and FOXO1 (1:100 dilution, Cell Signaling Technology) overnight at
$4{ }^{\circ} \mathrm{C}$ in a humidified chamber. Afterward, the sections were rinsed with PBS and incubated with an HRPconjugated goat anti-rabbit secondary antibody at $37{ }^{\circ} \mathrm{C}$ for $30 \mathrm{~min}$. HRP activity was detected using diaminobenzidine (Invitrogen, Carlsbad, CA, USA), and the sections were counterstained with hematoxylin. Nonspecific rabbit serum was used as a negative control, and mouse kidney (KLF12) and human breast carcinoma tissues (FOXO1) were employed as positive controls.

Measurement of KLF12 and FOXO1 protein expression in the endometrium samples was performed using ImagePro Plus System 6.0 (Media Cybernetics, Inc., Silver Spring, MD, USA) in a blinded fashion, without knowledge of the tissue source. Quantitative analysis was performed according to the software's instructions. The representative objective protein staining intensity (indicating the relative expression level) was determined according to the mean and integrated optical density (IOD) of the digital image $(\times 400)$. Signal density data for the tissue areas were obtained from five randomly selected fields of view and subjected to statistical analysis. 


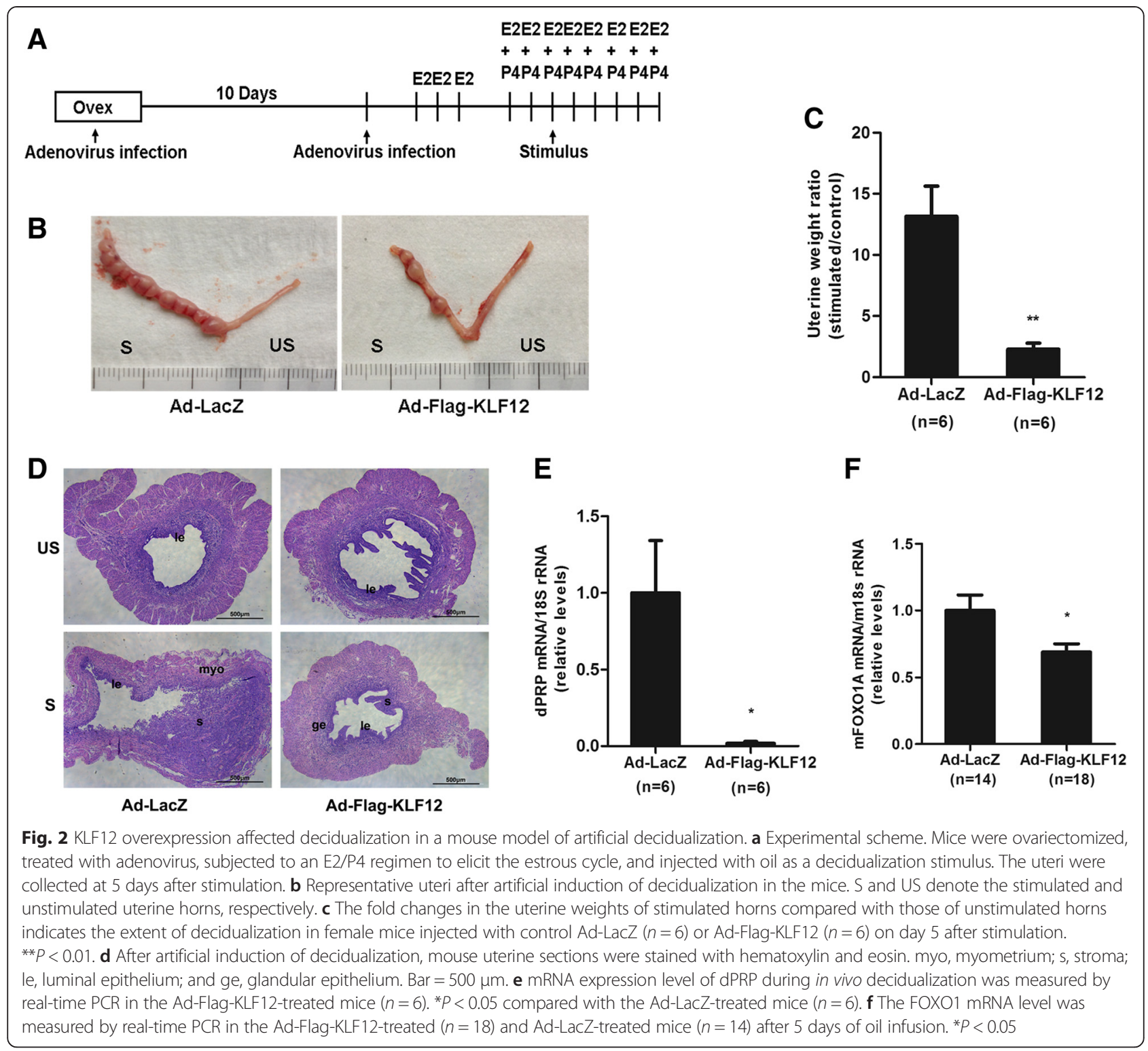

\section{Plasmid construction and reporter gene assays}

The wild-type FOXO1 promoter sequence, which spans from -3050 to $-2051 \mathrm{bp}$ relative to the transcription start site (Promoter ID: 11424), was amplified by PCR from hESC genomic DNA using the following primers: 5'-GGCCGGTACCTTACAGGATATTTATAATTTT-3' and 5'-AGAAAAGCTTGCCTTGCCTTCATTTCTAT TC-3'. The PCR product was cloned into a pGL3-basic luciferase reporter plasmid (Promega, USA).

Preconfluent (70\%) hESCs in 12-well plates were infected with Ad-Flag-KLF12 for $8 \mathrm{~h}$ and then transfected with $0.6 \mu \mathrm{g}$ FOXO1-Luc plasmid/well using Lipofectamine 2000 (Life Technologies, USA). After $6 \mathrm{~h}$, the culture medium was changed to DMEM-F12 (Invitrogen, Carlsbad, CA) with or without 8-Br-cAMP and medroxyprogesterone acetate (MPA). The cells were incubated for an additional $48 \mathrm{~h}$ and then harvested for preparation of cell extracts. Luciferase activity was measured with a Luciferase Assay System (Promega, Madison, USA), in which Renilla luciferase plasmids were cotransfected as controls to standardize the transcription efficiency. These assays were performed using a Centro XS3 LB 960 luminometer (Berthold Technologies, BW, Germany) according to the manufacturer's protocol.

\section{Chromatin immunoprecipitation (ChIP)-PCR}

Purified DNA fragments were extracted from hESCs and used as templates for PCR and real-time PCR as described previously [14]. A negative control primer set targeting a sequence distal $(-7672$ to $-7473 \mathrm{bp})$ to the putative binding site was chosen to monitor binding specificity, with the following sequences: 5 '-ACCTGC 
Table 1 Demographic details of the participants in the study of endometrial KLF12 expression

\begin{tabular}{llll}
\hline Fertile & FER $(n=14)$ & $\mathrm{RPL}(n=15)$ & $\mathrm{P}$ \\
\hline Age (years) & $29.5 \pm 3.8$ & $32.1 \pm 4.3$ & $\mathrm{~ns}$ \\
Body mass index $\left(\mathrm{kg} / \mathrm{m}^{2}\right)$ & $20.5 \pm 2.6$ & $22.2 \pm 5.9$ & $\mathrm{~ns}$ \\
P4 on hCG day $(\mathrm{pg} / \mathrm{mL})$ & $9.7 \pm 3.5$ & $9.3 \pm 3.2$ & $\mathrm{~ns}$ \\
Endometrial thickness $(\mathrm{mm})$ & $10.2 \pm 1.1$ & $10.0 \pm 1.8$ & $\mathrm{~ns}$ \\
No. of transferred embryos & $2 \pm 0$ & $6.5 \pm 2.6$ & $\mathrm{~s}$ \\
\hline
\end{tabular}

The data are presented as the mean \pm SD unless otherwise indicated. A $P<0.05$ was considered significant

P4 Serum progesterone concentration

TGACCCAGTGACTC-3' and 5' -AGGCTGAGGCAGGAGAATG-3' (-2766 to -2568 bp). The fold enrichment was calculated as the $2^{-\Delta \Delta C T}$ value and is presented relative to LacZ (after normalization to the input). Annealing was performed at $57{ }^{\circ} \mathrm{C}$, and the PCR products were electrophoresed on $2 \%$ agarose gels and visualized by GoldView staining.

\section{Avidin-biotin conjugate DNA precipitation (ABCD) assay} Double-stranded oligonucleotides were designed based on the FOXO1 promoter sequence ( -2637 to $-2601 \mathrm{bp}$ ). The 5 '-end of the sense strand was biotinylated, and a mutation was introduced (CAGTGGG to CACAAAG) to remove the specific binding site for KLF12. The following primers were designed: human FOXO1 wildtype: 5'-biotin-TCAGGCTGGAGTGCAGTGGGGCGATCTTGGCTCCGC-3'; human FOXO1 wild-type reverse: 5'-GCGGAGCCAAGATCGCCCCACTGCACTC CAGCCTGA-3'; and human FOXO1 mutant: 5'-biotinTCAGGCTGGAGTGCACAAAGGCGATCTTGGCTCC GC-3'. hESCs were infected with Ad-LacZ and Ad-FlagKLF12 (25 MOI) for $48 \mathrm{~h}$ and then incubated with 8-BrcAMP and MPA for an additional $48 \mathrm{~h}$. Cell extracts were harvested and lysed in RIPA buffer. Each double-stranded DNA sample (500 pmol) was incubated with $500 \mu \mathrm{g}$ of cell extract at $4{ }^{\circ} \mathrm{C}$ for $2-4 \mathrm{~h}$, and the protein complexes were pulled down using streptavidin agarose beads (Sigma) in binding buffer (10 mM Tris, pH 8.0; $150 \mathrm{mM} \mathrm{NaCl} ; 0.5 \%$ Triton X-100; $0.5 \mathrm{mM}$ DTT; $0.5 \mathrm{mM}$ EDTA; $10 \%$ glycerol; and $20 \mu \mathrm{g} / \mathrm{mL}$ poly $[\mathrm{dI}-\mathrm{dC}]$ ) containing a protease inhibitor cocktail. The proteins were eluted, separated by SDS-PAGE, and then probed with an anti-Flag-HRP antibody.

\section{Statistical analysis}

Unless stated otherwise, the numerical data are presented as the mean \pm SD of at least three independent experiments. Student's t-test was used to determine the statistical significance between two groups. ANOVA with Bonferroni's correction was employed for multiple comparisons. Pearson correlation analysis was used to assess the relationship between FOXO1 and KLF12. Differences at $P<0.05$ were considered significant.

\section{Results \\ KLF12 overexpression in the uterus decreased the embryo implantation rate}

Mouse embryo implantation occurs at midnight on 3.5 dpc, as determined based on preimplantation ovarian steroid profiles [21]. The uterine lumens of ICR mice were injected with a KLF12-expressing adenovirus (AdFlag-KLF12) or Ad-LacZ on 1.5 dpc (Fig. 1a), and the implantation status was then examined on $4.5 \mathrm{dpc}$ to determine whether KLF12 was a critical regulator of embryo implantation. Adenovirus-mediated overexpression of KLF12 markedly increased Flag-KLF12 fusion protein expression (Fig. 1d). The KLF12-overexpressing mice had fewer implantation sites $(6.18 \pm 1.03, n=11)$ compared with the Ad-LacZ group $(10.27 \pm 0.78, n=11)$ (Fig. 1b and c), suggesting that KLF12 blocked embryonic implantation.

\section{Increased KLF12 expression in the mouse uterus repressed uterine decidualization}

In a previous study, we demonstrated that KLF12 is a novel negative regulator of the decidual reaction and that KLF12 overexpression in hESCs significantly represses their morphological and biochemical transformation [14]. Based on the observations shown in Fig. 1, we hypothesized that impaired decidualization may cause KLF12induced implantation failure; thus, we investigated decidualization in the hormone-primed uteri of ovariectomized mice treated with a well-established regimen of E2 and P4 (Fig. 2a). As expected, morphological analysis of Ad-LacZ control mice revealed thicker uterine horns, indicating the presence of pronounced deciduoma within 5 days after artificial stimulation (Fig. 2b, left). In contrast, the uteri of KLF12-overexpressing mice contained thinner and fewer pseudopregnant sacs under identical conditions (Fig. 2b, right). As shown in Fig. 2c, the fold changes in the uterine weights of oil-stimulated horns compared with those of control horns indicated that the extent of decidualization was markedly reduced in the KLF12-overexpressing females. Morphometric analysis of uterine tissue sections further demonstrated that the stimulated Ad-LacZ horns were packed with decidual cells, while the KLF12overexpressing horns contained a markedly reduced number of decidual cells (Fig. 2d). Consistent with these results, the mRNA expression of dPRP, which is a marker of mouse decidualization [22], was significantly reduced by almost 50-fold in the uteri of the KLF12-overexpressing mice (Fig. 2e). Accordingly, increased uterine KLF12 expression was associated with severe impairment of decidualization. Additionally, FOXO1 mRNA was highly expressed in the control uteri but was down-regulated 
(reduced by $>30 \%$ ) in the uteri from the KLF12overexpressing mice (Fig. 2f).

\section{Elevated KLF12 level led to a decrease in FOXO1 expression}

To better understand the mechanism by which FOXO1 expression is regulated, we examined its expression following KLF12 up-regulation in hESCs in vitro. As shown in Fig. $3 \mathrm{a}$ and $\mathrm{b}$, infection of hESCs with Ad-Flag-KLF12 $(\mathrm{MOI}=25$ and 50$)$ for $48 \mathrm{~h}$ markedly increased KLF12 mRNA expression (by greater than 110-fold and 200fold, respectively). Importantly, KLF12 overexpression in hESCs resulted in significant dose-dependent decreases in FOXO1 mRNA (Fig. 3c) and protein (Fig. 3d) expression in response to 8-Br-cAMP and MPA.

\section{KLF12 directly repressed FOXO1 transcription}

We identified a conserved KLF12 binding element (CAGTGGG) within the FOXO1 core promoter region similar to a human FOXO1 promoter sequence deposited in transcriptional regulatory element database (Promoter ID: 11424, Fig. 4a). Exogenous hormone-induced decidual transformation increased FOXO1A promoter activity, as evidenced by luciferase reporter assays, and this increase was repressed by KLF12 overexpression (Fig. 4b). Next, ChIP-PCR assays showed that KLF12 bound to the FOXO1 promoter region $(-2637$ to $-2601 \mathrm{bp}$ ), which contains the conserved CAGTGGG element; however, no PCR product was obtained from the negative control region (-7672 to -7473 bp; Fig. 4c, top). Quantitative ChIP revealed that the primers were effectively (greater than 35-fold) amplified from FlagKLF12 protein immunoprecipitates but not from LacZ control immunoprecipitates (Fig. 4c, bottom). Moreover, Flag-tagged KLF12 protein strongly bound to the FOXO1 promoter containing the CAGTGGG sequence but not to the mutated probe (Fig. 4d). These data indicate that FOXO1 is a novel target gene of KLF12.

\section{KLF12 and FOXO1 expression was aberrant in the endometria of patients with RIF}

Because the decidual response of hESCs is disrupted in patients with RIF [23], we further assessed the expression of KLF12 and FOXO1 in midsecretory endometrial samples from women with RIF (Table 1). The endometrial KLF12 transcript and protein levels were higher (greater than 2-fold) in women with RIF compared with those in fertile controls (Fig. 5a, c and d). Conversely, expression of FOXO1 was, as expected, significantly reduced by $50 \%$ in the women with RIF compared with that in the fertile controls (Fig. 5b, c and e). The protein level of FOXO1 was moderately negatively correlated with that of KLF12 $(r=-0.4272395, P=0.04202)$. Immunohistochemical analysis revealed that the KLF12 and FOXO1 protein levels were higher and lower, respectively, in the endometria of patients with RIF compared with those in the endometria of the fertile controls, particularly in the stromal compartment (Fig. 5g). We quantified the KLF12 and FOXO1 expression densities in the endometrium using Image-Pro Plus System 6.0 image

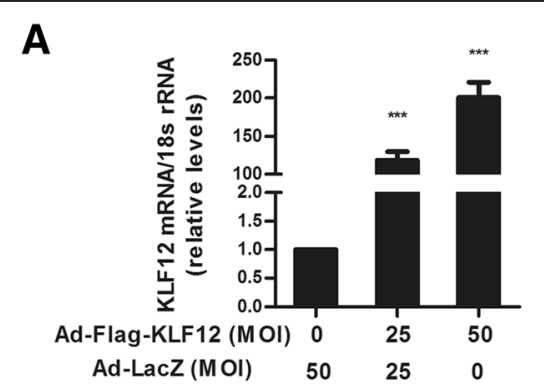

B

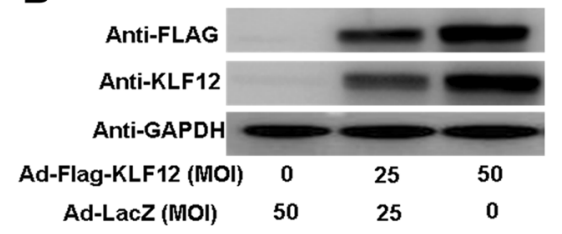

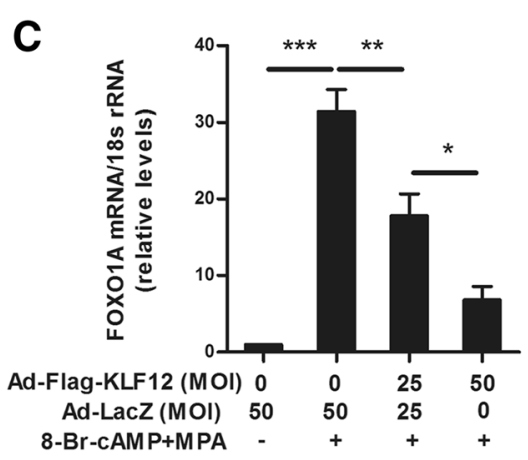

D

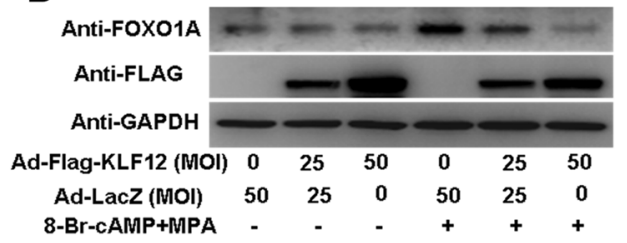

Fig. 3 Elevated KLF12 level led to decreased FOXO1 expression. hESCs were infected with adenovirus expressing either LacZ or KLF12 for $48 \mathrm{~h}$ at the indicated MOI. The KLF12 transcript copy number (a) and its protein expression (b) were determined by real-time PCR and Western blotting $(n=3) .{ }^{* * *} P<0.001$ compared with Ad-LacZ alone. hESCs were infected with Ad-LacZ or Ad-Flag-KLF12 at the indicated MOI for $48 \mathrm{~h}$ and were then treated with 8-Br-CAMP and MPA for another 2 days; subsequently, FOXO1 mRNA (c) and protein (d) levels were measured by real-time PCR and Western blotting, respectively $(n=3)$. ${ }^{*} P<0.05 ;{ }^{* *} P<0.01$; and ${ }^{* * *} P<0.001$ 


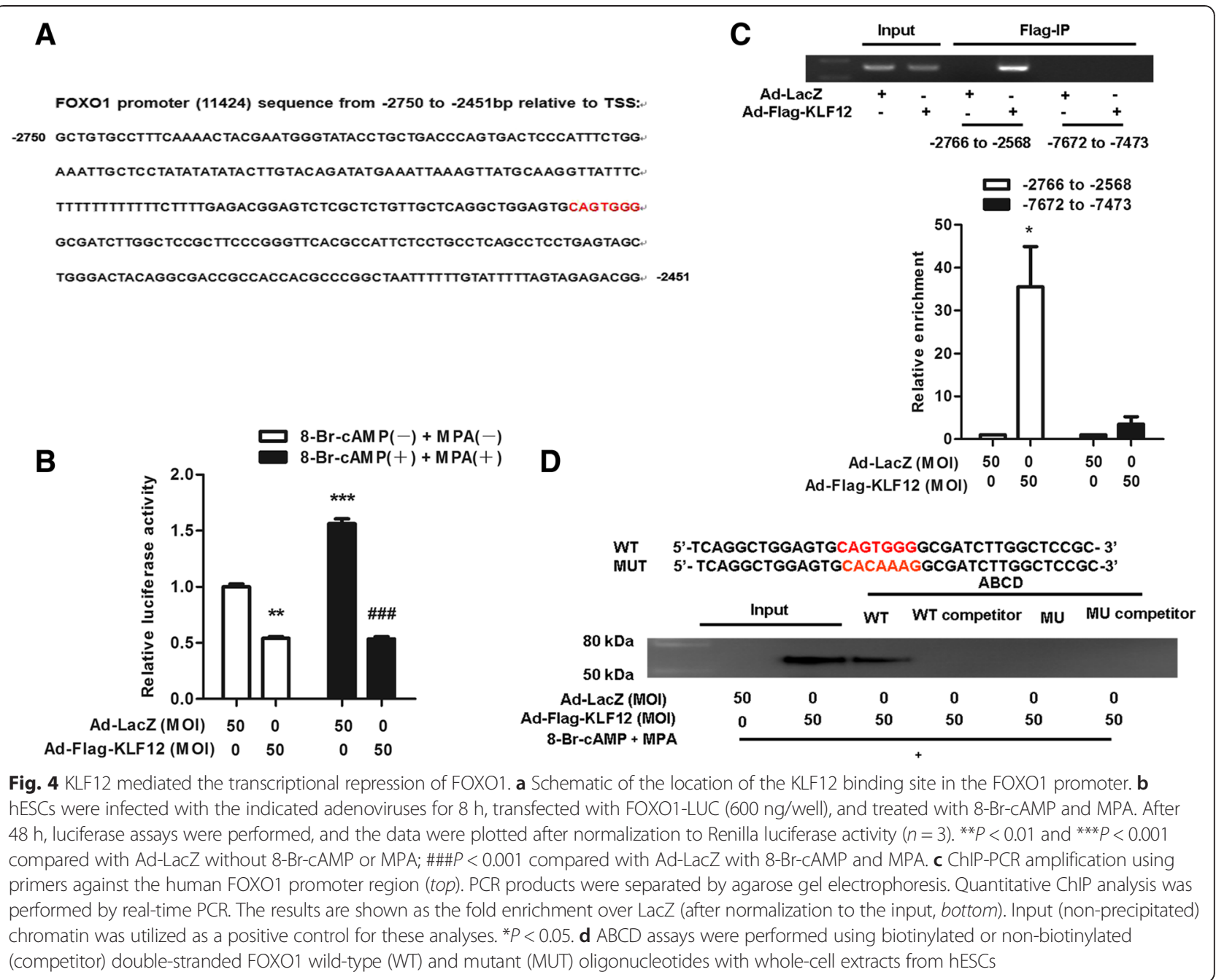

analysis software (Table 2). The IOD of KLF12 for the fertile controls was $232,246 \pm 59,354$, while that for the patients with RIF was $357,937 \pm 86,246$; in addition, the mean KLF12 expression density of the patients with RIF $(0.38 \pm 0.05)$ was higher than that of the controls $(0.31 \pm$ 0.06). The results also showed that the IOD of FOXO1 for the normal controls $(609,115 \pm 198,330)$ was significantly higher than that for the patients with RIF $(285,440 \pm 117,645)$. The mean densities for the fertile and RIF individuals were $0.34 \pm 0.08$ and $0.27 \pm 0.05$, respectively. The results of IHC assay shown in Fig. $5 \mathrm{~g}$ demonstrate that FOXO1-immunopositive epithelial cells could still be detected in the patients with RIF. We further calculated the level of FOXO1 expression in GE cells. The IOD was $178,465 \pm 88,230$ (FER, fertile controls) vs. $271,035 \pm 119,150$ (RIF), and the mean density was $0.23 \pm 0.06$ (FER) vs. $0.20 \pm 0.04$ (RIF) (Table 3). In GE cells, the IOD of KLF12 for the RIF group was higher than that for the normal group. These results suggested that increased KLF12 expression in the stromal compartment, together with decreased FOXO1 expression, contributed to the impaired decidualization observed in the women with RIF.

\section{Discussion}

This study has verified the important role of KLF12 in the induced decidualization in mice and is the first report of a putative molecular mechanism for the transcriptional repression of FOXO1 in hESCs. KLF12 belongs to the zinc finger-containing transcription factor family, which contains key mediators of endocrine/metabolic processes that play emerging key roles in human reproductive and uterine diseases $[15,24]$. For example, KLF11 is highly expressed in human urogenital tissues, and aberrant KLF11 expression has been linked to the pathogeneses of common uterine diseases, such as endometriosis and leiomyoma [25, 26]. Moreover, loss of coregulation between KLF9 and PR may underlie the pathogenesis of endometriosis [27]. Furthermore, KLF9 deficiency in the uterus has been shown to promote the establishment of ectopic 


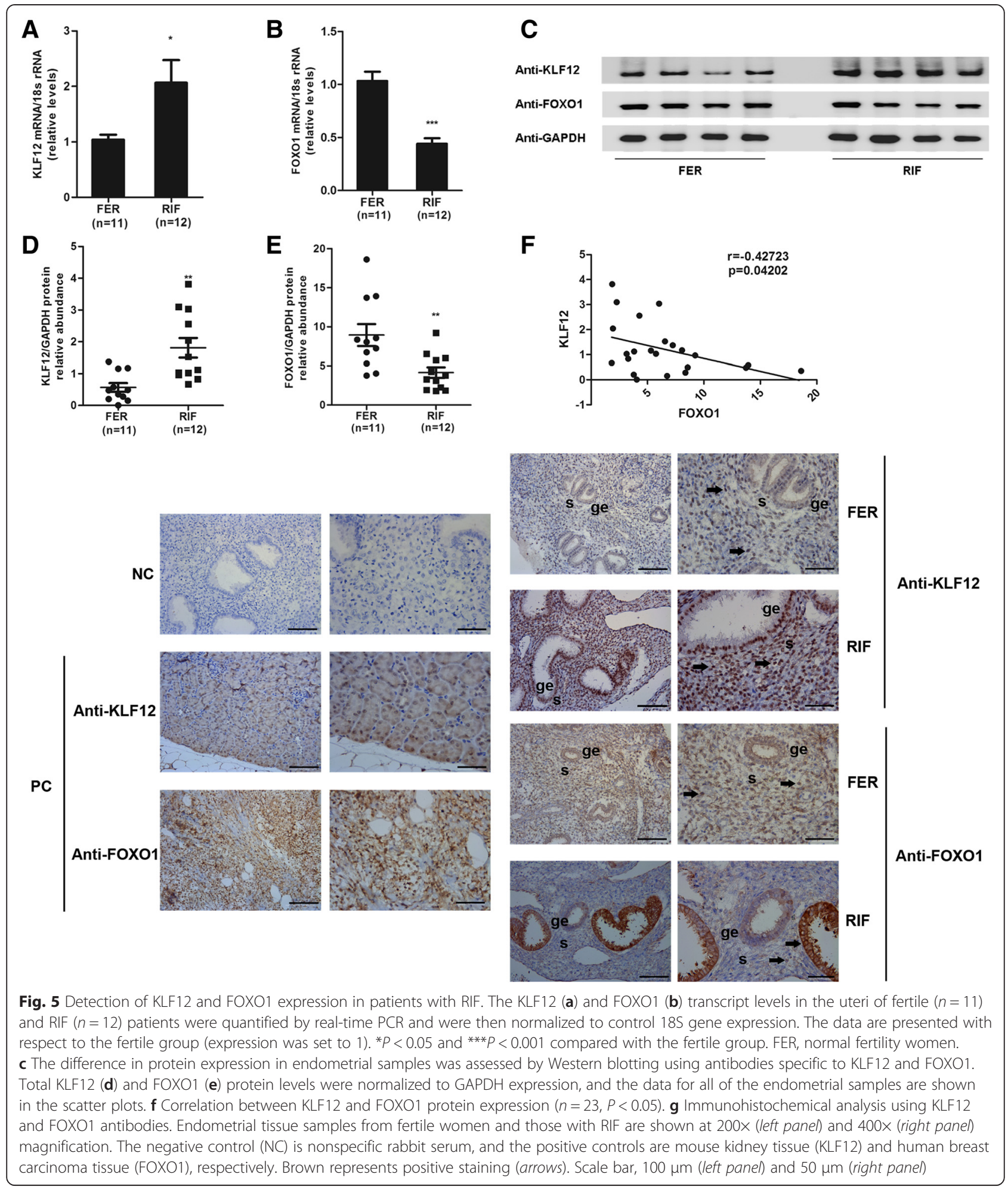

lesions in a mouse model of endometriosis [28]. Finally, KLF13-null lesions contribute to defective steroid hormone receptor signaling in the pathology of endometriosis [29], and KLF15 negatively regulates E2-induced epithelial cell proliferation, which is associated with endometriosis and endometrial cancer [30]. All of these reproductive diseases in which KLFs have been implicated are associated with impaired decidualization. Similarly, in this study, 
Table 2 The means and integrated optical densities of KLF12 and FOXO1 expression in endometrial tissue

\begin{tabular}{llll}
\hline Fertile & FER $(n=6)$ & $\operatorname{RPL}(n=6)$ & $\mathrm{P}$ \\
\hline $\begin{array}{l}\text { Mean density of KLF12 } \\
\text { expression }\end{array}$ & $0.31 \pm 0.006$ & $0.38 \pm 0.05$ & $<0.0001$ \\
KLF12 (IOD) & $232,246 \pm$ & $357,937 \pm$ & $<0.0001$ \\
& 59,354 & 86,246 & \\
Mean density of FOXO1 & $0.34 \pm 0.08$ & $0.27 \pm 0.05$ & 0.0403 \\
expression & & & \\
FOXO1 (IOD) & $609,115 \pm$ & $285,440 \pm$ & 0.0014 \\
& 198,330 & 117,645 & \\
\hline
\end{tabular}

The data are presented as the mean \pm SD. A $P<0.05$ was considered significant, vs. normal fertility women

increased KLF12 expression led to a severe reduction in endometrial decidualization and blockage of embryo implantation in the endometrium.

KLF12 (AP-2rep) was first identified based on its ability to bind to and down-regulate an important transcription factor, AP2 $\alpha$ [31]. Shen et al. found that PRL is a target gene of KLF12, which directly binds to the CAGTGGG sequence in the PRL promoter [14]. In the present study, we similarly identified a novel translational target of KLF12 in endometrial biology. FOXO1 is essential for female reproduction and is markedly induced upon decidualization by both the PKA/cAMP pathway and ligand-activated nuclear PR [32]. FOXO1 physically associates with HOXA10 or PR to modulate the expression of decidua-specific genes, such as insulinlike growth factor-binding protein 1 (IGFBP1) [33, 34]. Furthermore, KLF12 not only transcriptionally represses PRL expression but also reduces the IGFBP1 protein level during decidualization; however, the mechanism by which this regulation occurs remains unclear [14]. The KLF12-mediated repression of FOXO1 expression is potentially the primary cause of impaired decidualization and subsequent implantation failure. Because FOXO1 directly regulates IGFBP1 but KLF12 does not, the mechanism underlying the KLF12-mediated inhibition of IGFBP1 expression via FOXO1 during decidualization must be further addressed.

Table 3 The area and integrated optical density of FOXO1 expression in GE of endometrium

\begin{tabular}{llll}
\hline Fertile & FER $(n=6)$ & $\operatorname{RPL}(n=6)$ & $\mathrm{P}$ \\
\hline $\begin{array}{l}\text { Mean density of KLF12 } \\
\text { expression }\end{array}$ & $0.12 \pm 0.04$ & $0.15 \pm 0.05$ & 0.0282 \\
KLF12 (IOD) & $71,421 \pm$ & $134,775 \pm$ & 0.0021 \\
& 63,423 & 87,047 & \\
Mean density of FOXO1 & $0.23 \pm 0.06$ & $0.20 \pm 0.04$ & 0.0012 \\
expression & & & \\
FOXO1 (IOD) & $178,465 \pm$ & $271,035 \pm$ & 0.0290 \\
& 88,230 & 119,150 & \\
\hline
\end{tabular}

The data are presented as the mean \pm SD. A $P<0.05$ was considered significant, vs. normal fertility women
Although assisted reproductive technologies have markedly reduced the incidence of infertility, 45-60\% of patients undergoing assisted reproduction do not experience a live birth after 3 treatment cycles [35]. Endometrial receptivity is responsible for RIF in approximately two-thirds of affected women [36], and stromal decidualization is considered to be important for endometrial receptivity [4].

As suggested by immunohistochemical analysis of the endometrium of women with RIF, KLF12 expression is elevated in stromal cells as well as in the GE. Interactions between the blastocyst and epithelia and those between epithelial cells and stromal cells are vital to successful implantation [37, 38]. Paracrine signals from the blastocyst result in the production of various hormones and cytokines (e.g., chorionic gonadotropin and interleukin-1) that facilitate attachment and maintenance of pregnancy $[39,40]$. After blastocyst attachment, luminal epithelial cells transmit signals to mediate the proliferation and differentiation of the endometrium and to elicit changes in vascular permeability and embryo migration across the epithelium [41, 42]. The GE, another part of the endometrial epithelium, is embedded within the stromal bed. Gland-derived signaling influences stromal function in a paracrine manner [43]. Many products secreted from the GE, such as leukemia inhibitory factor, appear to support nascent embryos and normal stromal decidualization during implantation [44, 45]. Aberrant KLF12 expression in the GE may interfere with crosstalk between the epithelium and stroma, causing hESC decidualization defects. Furthermore, as an antiinflammatory regulator, KLF12 is down-regulated in patients with aneurysm [46], and decidualization and embryo implantation are usually considered to involve proinflammatory responses. The higher epithelial KLF12 expression observed during implantation in women with RIF indicates that the product of this gene may influence the immuno-inflammatory reaction in the uterine epithelium. Interestingly, in our study, the mean density of FOXO1 in the GE of the control group was slightly higher than that in the GE of the RIF group, while the IOD was weaker in the control group than that in the RIF group. These differences were associated with a significant change in the number of GE cells, as well as slightly altered KLF12 expression in these cells. On the other hand, FOXO1 is required for programmed apoptosis of the epithelium and for safeguarding endometrial homeostasis during the reproductive phase [11]. Additionally, FOXO1 may be regulated by factors other than KLF12, such as miRNAs, in endometrial epithelial cells [47].

KLF12 is involved in defective decidualization in patients with RIF; however, the mechanism by which it is up-regulated remains unclear. Compromised P4 signaling might be responsible for the abnormal endometrial 
gene expression observed in women with RIF. Thus, the outcomes of RIF may be ascribed to abnormal P4sensitive maternal signaling rather than to the amount of progesterone, given the similar levels of secreted progesterone in the patients and controls observed in this study. MPA is a synthetic progesterone that interacts with PR to promote differentiation of the uterine endometrium, and in our study, MPA repressed KLF12 expression in hESCs in a time-dependent manner (data not shown). Rubel et al. reported that many members of the KLF family, such as KLFs 3, 4, 7, and 12, are candidate mediators of PR function in P4-responsive target tissues [48]. Therefore, KLF12, a potential P4-regulated gene, may be up-regulated by compromised $\mathrm{P} 4$ signaling in the endometria of patients with RIF.

This study constitutes the first functional analysis of the role of KLF12 in endometrial decidualization and embryo implantation in vivo. To prevent traumatic adenovirus infection of the uterine horns, a mouse uterine model with a conditional KLF12 knock-in will be employed in future studies to unravel the physiological role and mechanism of action of KLF12 in pregnancy.

\section{Conclusions}

Taken together, our results indicate that KLF12, by negatively regulating FOXO1 expression, contributes to improper stromal decidualization and reduced embryo implantation. These findings may inspire the development of new therapeutic regimens for patients with RIF and disrupted decidualization.

\section{Additional file}

Additional file 1: Figure S1. Cellular immunohistochemical staining of hESCs. (DOCX 617 kb)

\section{Abbreviations \\ C/EBP $\beta$ : CCAAT/enhancer-binding protein $\beta$; E2: Estrogen; FER: Fertility; FOXO1: Forkhead box class O1; FOXOs: O subclass of the forkhead family of transcription factors; GE: Glandular epithelium; hESC: Human endometrial stromal cell; HRP: Horseradish peroxidase; IGFBP1: Insulin-like growth factor-binding protein 1; IOD: Integrated optical densities; IS: Implantation site; IVF: In vitro fertilization; KLF5: Krüppel-like factor 5; KLF12: Krüppel-like factor 12; MPA: Medroxyprogesterone acetate; MUT: Mutant; NC: Negative control; P4: Progesterone; PC: Positive control; PR: Progesterone receptor; PRL: Prolactin; PRP: Prolactin-related protein; RIF: Repeated implantation failure; RPL: Recurrent pregnancy loss; S: Stimulated uterine horns; US: Unstimulated uterine horns; WT: Wild type.}

\section{Competing interests}

The authors declare that they have no competing interests.

\section{Authors' contributions}

$H S, G Y$ and XDZ were responsible for the conception and design of the study. HZ, JC, YJ, QZ, CK, and JX were responsible for acquisition of data. HZ, $J C, J X, L D, Z D, X Z, H S$ and GY performed data analysis and drafted the manuscript. All authors participated in interpretation of the findings. $\mathrm{HZ}, \mathrm{HS}$ and GY revised and commented on the draft. All authors read and approved the final version of the manuscript.

\section{Acknowledgements}

This work was supported by the National Natural Science Foundation of China (No. 81370683, 81170570 and 81200127), special grants for principal investigators from the Health Department of Jiangsu Province (No. LJ201102 and RC2011005) and the Six Top Talents Program of Jiangsu Province, PR China (No. 2012-WSN-005).

\section{Author details}

'Reproductive Medicine Center, Drum Tower Clinic Medical College of Nanjing Medical University, Nanjing 210029, Jiangsu, China. ${ }^{2}$ College of Science Isotope Laboratory, Nanjing Agricultural University, Nanjing 210095, Jiangsu, China. ${ }^{3}$ Department of Obstetrics and Gynecology, Reproductive Medicine Center, Nanjing Drum Tower Hospital, Nanjing University Medical School, Nanjing 210008, Jiangsu, China.

Received: 9 March 2015 Accepted: 18 July 2015

Published online: 30 July 2015

\section{References}

1. Gellersen B, Brosens JJ. Cyclic decidualization of the human endometrium in reproductive health and failure. Endocr Rev. 2014;35:851-905.

2. Salamonsen LA, Dimitriadis E, Jones RL, Nie G. Complex regulation of decidualization: a role for cytokines and proteases-a review. Placenta. 2003;24(Suppl A):S76-85.

3. Salker M, Teklenburg G, Molokhia M, Lavery S, Trew G, Aojanepong T, et al. Natural selection of human embryos: impaired decidualization of endometrium disables embryo-maternal interactions and causes recurrent pregnancy loss. PLoS One. 2010;5:e10287.

4. Salamonsen LA, Nie G, Hannan NJ, Dimitriadis E. Society for Reproductive Biology Founders' Lecture 2009. Preparing fertile soil: the importance of endometrial receptivity. Reprod Fertil Dev. 2009;2009(21):923-34.

5. Kobayashi $\mathrm{H}$, Uekuri $\mathrm{C}$, Shigetomi $\mathrm{H}$. Towards an understanding of the molecular mechanism of endometriosis: unbalancing epithelial-stromal genetic conflict. Gynecol Endocrinol. 2014;30:7-15.

6. Brosens JJ, Pijnenborg R, Brosens IA. The myometrial junctional zone spiral arteries in normal and abnormal pregnancies: a review of the literature. Am J Obstet Gynecol. 2002;187:1416-23.

7. Ramathal CY, Bagchi IC, Taylor RN, Bagchi MK. Endometrial decidualization: of mice and men. Semin Reprod Med. 2010;28:17-26.

8. Estella C, Herrer I, Moreno-Moya JM, Quinonero A, Martinez S, Pellicer A, et al. miRNA signature and Dicer requirement during human endometrial stromal decidualization in vitro. PLoS One. 2012;7:e41080.

9. Lim H, Ma L, Ma WG, Maas RL, Dey SK. Hoxa-10 regulates uterine stromal cell responsiveness to progesterone during implantation and decidualization in the mouse. Mol Endocrinol. 1999;13:1005-17.

10. Mantena SR, Kannan A, Cheon YP, Li Q, Johnson PF, Bagchi IC, et al. C/ EBPbeta is a critical mediator of steroid hormone-regulated cell proliferation and differentiation in the uterine epithelium and stroma. Proc Natl Acad Sci U S A. 2006;103:1870-5.

11. Brosens JJ, Wilson MS, Lam EW. FOXO transcription factors: from cell fate decisions to regulation of human female reproduction. Adv Exp Med Biol. 2009;665:227-41.

12. Sun X, Zhang L, Xie H, Wan H, Magella B, Whitsett JA, et al. Kruppel-like factor 5 (KLF5) is critical for conferring uterine receptivity to implantation. Proc Natl Acad Sci U S A. 2012;109:1145-50.

13. Simmen RC, Pabona JM, Velarde MC, Simmons C, Rahal O, Simmen FA. The emerging role of Kruppel-like factors in endocrine-responsive cancers of female reproductive tissues. J Endocrinol. 2010;204:223-31.

14. Shen $X$, Hu Y, Jiang $Y$, Liu $H$, Zhu L, Jin $X$, et al. Kruppel-like factor 12 negatively regulates human endometrial stromal cell decidualization. Biochem Biophys Res Commun. 2013;433:11-7.

15. Simmen RC, Heard ME, Simmen AM, Montales MT, Marji M, Scanlon S, et al. The Kruppel-like factors in female reproductive system pathologies. J Mol Endocrinol. 2015;54:R89-101.

16. Schuierer M, Hilger-Eversheim K, Dobner T, Bosserhoff AK, Moser M, Turner $\mathrm{J}$, et al. Induction of AP-2alpha expression by adenoviral infection involves inactivation of the AP-2rep transcriptional corepressor CtBP1. J Biol Chem. 2001;276:27944-9.

17. Wang W, Li Q, Bagchi IC, Bagchi MK. The CCAAT/enhancer binding protein beta is a critical regulator of steroid-induced mitotic expansion of uterine stromal cells during decidualization. Endocrinology. 2010;151:3929-40. 
18. Coughlan C, Ledger W, Wang Q, Liu F, Demirol A, Gurgan T, et al. Recurrent implantation failure: definition and management. Reprod Biomed Online. 2014;28:14-38

19. Jiang $Y$, Hu Y, Zhao J, Zhen $X$, Yan G, Sun H. The orphan nuclear receptor Nur77 regulates decidual prolactin expression in human endometrial stromal cells. Biochem Biophys Res Commun. 2011;404:628-33.

20. Liu $H$, Jiang $Y$, Jin $X$, Zhu L, Shen $X$, Zhang Q, et al. CAPN 7 promotes the migration and invasion of human endometrial stromal cell by regulating matrix metalloproteinase 2 activity. Reprod Biol Endocrinol. 2013;11:64.

21. Yoshinaga K. A sequence of events in the uterus prior to implantation in the mouse. J Assist Reprod Genet. 2013;30:1017-22.

22. Gu Y, Soares MJ, Srivastava RK, Gibori G. Expression of decidual prolactin-related protein in the rat decidua. Endocrinology. 1994;135:1422-7.

23. Fatemi HM, Popovic-Todorovic B. Implantation in assisted reproduction: a look at endometrial receptivity. Reprod Biomed Online. 2013;27:530-8.

24. Zheng Y, Tabbaa ZM, Khan Z, Schoolmeester JK, El-Nashar S, Famuyide A, et al. Epigenetic regulation of uterine biology by transcription factor KLF11 via posttranslational histone deacetylation of cytochrome p450 metabolic enzymes. Endocrinology. 2014;155:4507-20.

25. Daftary GS, Zheng Y, Tabbaa ZM, Schoolmeester JK, Gada RP, Grzenda AL, et al. A novel role of the Sp/KLF transcription factor KLF11 in arresting progression of endometriosis. PLoS One. 2013;8:e60165.

26. Yin $\mathrm{P}$, Lin Z, Reierstad S, Wu J, Ishikawa H, Marsh EE, et al. Transcription factor KLF11 integrates progesterone receptor signaling and proliferation in uterine leiomyoma cells. Cancer Res. 2010;70:1722-30.

27. Pabona JM, Simmen FA, Nikiforov MA, Zhuang D, Shankar K, Velarde MC, et al. Kruppel-like factor 9 and progesterone receptor coregulation of decidualizing endometrial stromal cells: implications for the pathogenesis of endometriosis. J Clin Endocrinol Metab. 2012;97:E376-92.

28. Heard ME, Simmons CD, Simmen FA, Simmen RC. Kruppel-like factor 9 deficiency in uterine endometrial cells promotes ectopic lesion establishment associated with activated notch and hedgehog signaling in a mouse model of endometriosis. Endocrinology. 2014;155:1532-46.

29. Heard ME, Velarde MC, Giudice LC, Simmen FA, Simmen RC. Kruppel-like factor 13 deficiency in uterine endometrial cells contributes to defective steroid hormone receptor signaling but not lesion establishment in a mouse model of endometriosis. Biol Reprod. 2015;92(6):140. doi:10.1095/ biolreprod.115.130260.

30. Ray S, Pollard JW. KLF15 negatively regulates estrogen-induced epithelial cell proliferation by inhibition of DNA replication licensing. Proc Natl Acad Sci U S A. 2012;109:E1334-43.

31. Imhof A, Schuierer M, Werner O, Moser M, Roth C, Bauer R, et al. Transcriptional regulation of the AP-2alpha promoter by BTEB-1 and AP-2rep, a novel wt-1/egr-related zinc finger repressor. Mol Cell Biol. 1999;19:194-204.

32. Fan W, Li SW, Li WH, Wang Y, Gong Y, Ma QH, et al. FOXO1 expression and regulation in endometrial tissue during the menstrual cycle and in early pregnancy decidua. Gynecol Obstet Invest. 2012;74:56-63.

33. Kim JJ, Taylor HS, Akbas GE, Foucher I, Trembleau A, Jaffe RC, et al. Regulation of insulin-like growth factor binding protein-1 promoter activity by FKHR and HOXA10 in primate endometrial cells. Biol Reprod. 2003;68:24-30

34. Takano M, Lu Z, Goto T, Fusi L, Higham J, Francis J, et al. Transcriptional cross talk between the forkhead transcription factor forkhead box 01A and the progesterone receptor coordinates cell cycle regulation and differentiation in human endometrial stromal cells. Mol Endocrinol. 2007;21:2334-49.

35. Polanski LT, Baumgarten MN, Quenby S, Brosens J, Campbell BK, Raine-Fenning NJ. What exactly do we mean by 'recurrent implantation failure'? A systematic review and opinion. Reprod Biomed Online. 2014;28:409-23.

36. Ledee-Bataille N, Lapree-Delage G, Taupin JL, Dubanchet S, Frydman R, Chaouat G. Concentration of leukaemia inhibitory factor (LIF) in uterine flushing fluid is highly predictive of embryo implantation. Hum Reprod. 2002;17:213-8.

37. Srisuparp S, Strakova Z, Brudney A, Mukherjee S, Reierstad S, Hunzicker-Dunn M, et al. Signal transduction pathways activated by chorionic gonadotropin in the primate endometrial epithelial cells. Biol Reprod. 2003:68:457-64.

38. Pawar S, Hantak AM, Bagchi IC, Bagchi MK. Minireview: Steroid-regulated paracrine mechanisms controlling implantation. Mol Endocrinol. 2014;28:1408-22.
39. Cameo P, Srisuparp S, Strakova Z, Fazleabas AT. Chorionic gonadotropin and uterine dialogue in the primate. Reprod Biol Endocrinol. 2004;2:50.

40. Fazleabas AT, Kim JJ, Strakova Z. Implantation: embryonic signals and the modulation of the uterine environment-a review. Placenta. 2004:25 (Suppl A):S26-31.

41. Pawar S, Starosvetsky E, Orvis GD, Behringer RR, Bagchi IC, Bagchi MK. STAT3 regulates uterine epithelial remodeling and epithelial-stromal crosstalk during implantation. Mol Endocrinol. 2013;27:1996-2012.

42. Aplin JD. Embryo implantation: the molecular mechanism remains elusive. Reprod Biomed Online. 2006;13:833-9.

43. Zhang S, Lin H, Kong S, Wang S, Wang H, Armant DR. Physiological and molecular determinants of embryo implantation. Mol Aspects Med. 2013;34:939-80

44. Lessey BA. The role of the endometrium during embryo implantation. Hum Reprod. 2000;15 Suppl 6:39-50.

45. Bhatt $H$, Brunet $L J$, Stewart CL. Uterine expression of leukemia inhibitory factor coincides with the onset of blastocyst implantation. Proc Natl Acad Sci U S A. 1991;88:11408-12.

46. Nakaoka H, Tajima A, Yoneyama T, Hosomichi K, Kasuya H, Mizutani T, et al. Gene expression profiling reveals distinct molecular signatures associated with the rupture of intracranial aneurysm. Stroke. 2014;45:2239-45.

47. Tang T, Wong HK, Gu W, Yu MY, To KF, Wang CC, et al. MicroRNA-182 plays an onco-miRNA role in cervical cancer. Gynecol Oncol. 2013;129:199-208.

48. Rubel CA, Lanz RB, Kommagani R, Franco HL, Lydon JP, DeMayo FJ. Research resource: Genome-wide profiling of progesterone receptor binding in the mouse uterus. Mol Endocrinol. 2012;26:1428-42.

\section{Submit your next manuscript to BioMed Central and take full advantage of:}

- Convenient online submission

- Thorough peer review

- No space constraints or color figure charges

- Immediate publication on acceptance

- Inclusion in PubMed, CAS, Scopus and Google Scholar

- Research which is freely available for redistribution 